\title{
The effects of three levels of lick-contingent footshock on schedule-induced polydipsia
}

\author{
EDWARD P. GALANTOWICZ and GLEN D. KING \\ Auburn University, Auburn, Alabama 36830
}

\begin{abstract}
Nine food deprived female albino rats were divided into three shock groups with three subjects in each group. The subjects were subjected to five successive experimental phases utilizing baseline, intermittent schedule, and intermittent schedule plus contingent shock condition, with individual subject water intakes recorded for each 60-min daily session across all phases. During the experimental shock phase, Groups 1,2 , and 3 received $2.0,1.0$, and $0.1 \mathrm{~mA}$ postbellet lick-contingent shock, respectively. 2.0-mA shocks distupted emotionally induced drinking, whereas 1.0-mA shocks had no effect on emotionally induced drinking and $0.1-\mathrm{mA}$ shock facilitated drinking. These results have important implications for the consideration of using punishment techniques as treatments in the eradication of undesirable behaviors.
\end{abstract}

Falk (1961, 1964) has noted that experimentally naive albino rats responding for 45-mg food pellets on an intermittent reinforcement schedule (VI 1-min) ingested unusually large amounts of water. The rats' mean water intake for each daily experimental session $(3.17 \mathrm{~h})$ was 3.43 times as great as their usual, preexperimental home cage water intake over an entire 24 -h period. Since these same animals were also food deprived, the levels of water consumption were even more dramatic, since food deprivation has been shown to be associated typically with marked hypodipsia (Calvin \& Behan, 1954). Falk labeled this phenomenon "schedule-induced polydipsia."

Subsequent experimental investigation of schedule-induced polydipsia (SIP) has resulted in several theoretical attempts to explain the phenomenon adequately (Segal \& Oden, 1965). These explanations variously suggest that SIP involves such phenomena as timing behavior, thirst factors, adventitious reinforcement, and more recently, emotional arousal. While the bulk of research has focused on the first three proposed explanations for SIP, the latter notion of emotional arousal as being sufficient to elicit extraordinary drinking behavior in rats is not without experimental precedent (Levine, 1965; Moyer, 1965). The prominence of motivational/emotional states within SIP has been convincingly demonstrated (Falk, 1964; Roll, Schaeffer, \& Smith, 1969), and subsequent investigations using electric shock within the SIP paradigm appear to support these earlier positions (King, 1972, 1974; Segal \& Oden, 1969).

King (1974) offered a summary perspective of the research concerning emotional arousal in SIP. Various aversive conditions such as food deprivation and intermittent reinforcement included within the

This research was supported in part by Public Health Service Research Grants MH-08775, MH 12025, and the Auburn University Research Council. The experiment comprised the major author's Masters Thesis. Robert W. Schaeffer sponsors this paper and takes full editorial responsibility for its contents. Requests for reprints should be sent to Glen D. King. experimental design, suggests King, develop in the organism an emotional state that serves to energize that organism's most probable response. A rat's most probable response following food ingestion is to drink, and it is this drinking response that tends to be energized to excess within the SIP paradigm. If the high levels of drinking seen in SIP are, in fact, expressions of underlying emotional states, then changing the intensities of these states should show concommitant changes in the amounts of drinking. The results reported in King's (1974) study support this hypothesis.

The purpose of the present experiment was to determine the effect upon SIP brought about by the administration of lick-contingent footshock at varying levels of intensity. It was hypothesized that if a highly intensified emotional state, induced by the intermittent presentation of food, was responsible for the increased amounts of drinking elicited in typical SIP paradigms, the additional stress of electric shock plus intermittent scheduling of reinforcement would result in even greater fluid intakes. It was further suggested that these greater fluid intakes would tend to vary as a function of the different levels of shock utilized.

\section{Subjects}

\section{METHOD}

The subjects were nine experimental naive female albino rats approximately 120 days old and individually housed in Wahmann LC175-C75 home cages. During the experiment all subjects were maintained at $80 \%$ of their free-feeding body weights. Food pellets obtained during experimental sessions were supplemented as necessary with Purina rat chow in the home cages following each session. Water was freely available in both the home cage and the experimental chamber.

\section{Apparatus}

Three LeHigh Valley Electronics chambers (No. 132-02) were employed with the right lever removed and a drinking spout inserted in its place. Noyes $45-\mathrm{mg}$ sugarless pellets (cf., Christian \& Schaeffer, 1973) were delivered automatically via appropriate relay circuitry. The number of reinforcements and drinking spout licks were recorded on Gerbrands cumulative recorders, an 
Esterline-Angus digital operations recorder, and Foringer counters. Footshock was delivered using three BRS/LVE SGS-004 shock generator/scramblers. The lickometers were electromechanically disconnected for $.6 \mathrm{sec}$ during shock applications. Individual water intakes during each experimental session were determined by the difference between presession and postsession bottle weights.

\section{Procedure}

The experiment consisted of five phases: (1) Baseline 1; (2) Baseline 2; (3) experimental; (4) replication of Baseline 2; (5) replication of Baseline 1. Daily sessions in all five phases were $60 \mathrm{~min}$ in duration.

During Baseline 1, all subjects were magazine trained by placing 60 pellets in the magazine with the empty feeder operating on the schedule used in Phases $2-4$ as an added experimental control. Water was freely available during this and all subsequent phases.

In Baseline 2, all subjects received 60 pellets on a FT $60-\mathrm{sec}$ schedule. A FT schedule delivers pellets on a periodic schedule, independently of the organism's behavior. Following stabilization and prior to the experimental phase, subjects were assigned on the basis of individual mean water intake to one of three groups so that the mean water intake for each group was approximately equal. An analysis of variance on water intakes by groups was conducted which showed no significant differences between groups on water intakes.

Phase 3 was a replication of Baseline 2 with the addition of a FR lick contingent shock. Each subject in each group received a .5 -sec shock after the first 80 postpellet licks on the drink tube. Only one shock was delivered per interpellet interval to any given subject. The intensity of the shock was constant for subjects within a group, but different across groups with Group 1 receiving 2.0-mA shocks, Group 2 receiving $1.0-\mathrm{mA}$ shocks, and Group 3 receiving .1-mA shocks.

Phases 4 and 5 involved a replication of the conditions employed in Baselines 2 and 1, respectively.

Each phase of the experiment remained in ettect untu water intakes for each subject in each phase stablized. The criterion for stablization was met when the difference between the mean individual water intake for the last 3 days minus the mean water intake for the preceding 3 days did not exceed $25 \%$ of the mean water intake for the last 6 days.

\section{RESULTS AND DISCUSSION}

The mean water intakes across all subjects in each shock group for each experimental phase are presented in Figure 1. The data indicate that each shock group exhibited increases in water consumption in the schedule-induced polydipsia phase without shock (Phase 2) relative to the baseline phase (Phase 1). With the introduction of lick-contingent footshock in Phase 3, the groups appeared to alter their water intakes relative to Phase 2 according to the intensity of the shock.

Group 1, which received 2.0-mA lick-contingent shock, exhibited a sharp decrease in the amount of water consumed while Group 2 (1.0-mA shock) water intake remained essentially unchanged and Group 2 (.1-mA shock exhibited an increase in water consumption.

A surprising effect occurred when shock was discontinued in Phase 4. Water intake levels, rather than decreasing or remaining the same as might be expected, increased substantially for Group 1 . One explanation for this phenomenon is that the $2.0-\mathrm{mA}$ lick-contingent shock to the subjects in Group 1 increased emotional

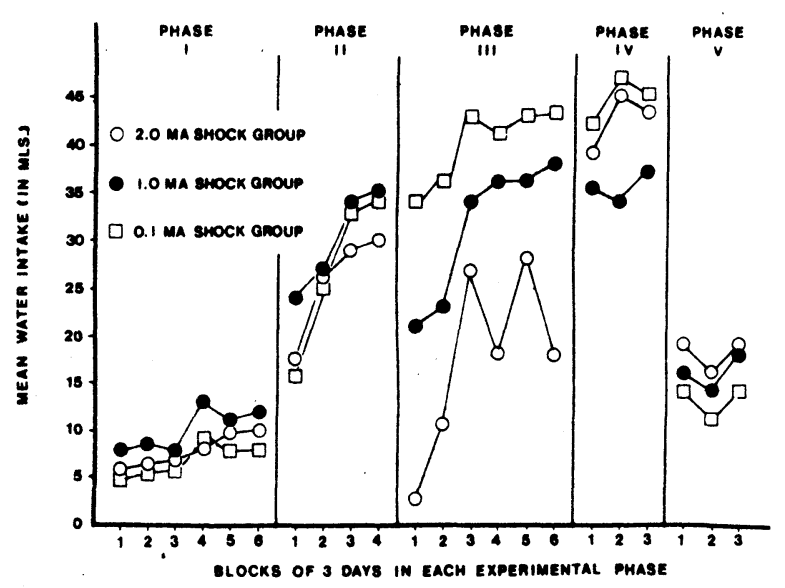

Figure 1. Mean water intakes for each shock group across all experimental phases.

arousal but was at the same time sufficiently intense to interfere with the continuance of the licking response. When shock was terminated in Phase 4 , thereby eradicating interference with the lick burst, the emotional arousal conditioned to the SIP situation by the repeated presentation of the shock in the previous phase resulted in increased water consumption.

In order to assess statistically the effects of experimental procedures on the consumption of water, an analysis of variance for a three factor mixed design with repeated measures on two factors was conducted. Results indicated that there was a significant difference in water consumption for all groups between phases $(p<.001)$. Scheffé multiple comparison tests were performed to determine the locus of significant differences. The results indicated that a comparison of Group 1 and Group 3 water intakes in Phase 3 was significant $(p<.05)$, and the comparisons of the amounts of water consumed across groups between all five experimental phases were significant $(\mathrm{p}<.01)$ with two exceptions: the comparisons of water intakes between Phases 1 and 5 and between Phases 2 and 3 were nonsignificant $(\mathrm{p}>.05)$.

These findings lend support to the notion that low levels of punishment (.1-mA shock) may actually facilitate rather than suppress an ongoing behavior presumed to be due in part to emotional arousal, while moderate levels of punishment (1.0-mA shock) have no appreciable effect on ongoing emotionally induced behaviors and high levels of punishment (2.0-mA shock) have a temporarily disruptive effect on such behaviors.

To determine the locus of effects on water consumption through institution of an intermittent pellet delivery schedule and subsequent lick-contingent shock, an analysis of the lick bursts for each group was conducted with results presented in Table 1. Each group's lick burst pattern is representative of the patterns emitted individually by each subject within that group.

The data for Group 1 (2.0-mA shock) indicate that 
Table 1

Group Lick Burst Lengths (Mean Number and Percent) Across the Last Three Days for Each Experimental Phase

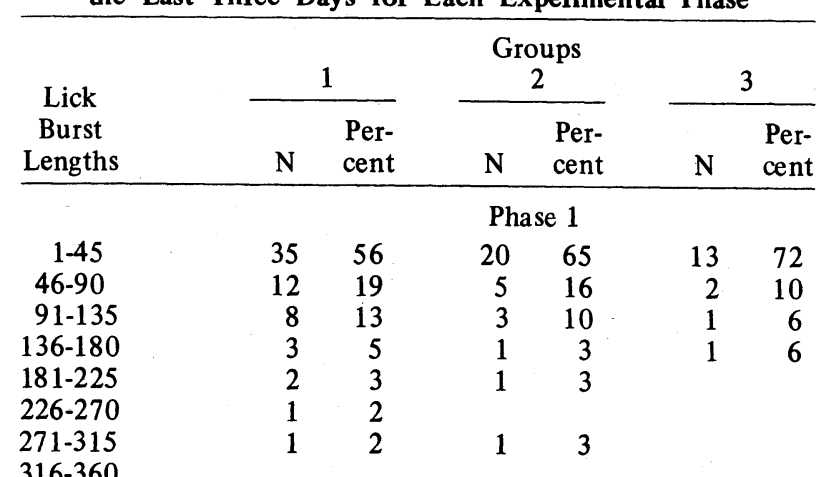

$316-360$

$361-405$

406-450

Over 450

Total

Median Length

62

40

$1-45$
$46-90$

91-135

136-180

$181-225$

226-270

271-315

$316-360$

$361-405$

406-450

Over 450

Total

58

Median Length $\quad 101$

$1-45$
$46-90$
$91-135$
$136-180$
$181-225$
$226-270$
$271-315$
$316-360$
$361-405$
$406-450$
Over 450

Total

Median Length

82

36

1-45

46-90

91-135

136-180

181-225

226-270

271-315

$316-360$

$361-405$

406-450

Over 450

Total

Median Length

110

$1-45$

$51 \quad 66$
$59 \quad 54$

$18 \quad 16$

$21 \quad 19$

$12 \quad 11$
70

Phase 3

1323

1120

$\begin{array}{ll}15 & 27 \\ 10 & 18\end{array}$

$\begin{array}{ll}2 & 4 \\ 1 & 2\end{array}$

$\begin{array}{ll}1 & 2 \\ 1 & 4\end{array}$

$\begin{array}{rr}6 & 13 \\ 13 & 27 \\ 11 & 23 \\ 9 & 19 \\ 3 & 6 \\ 1 & 2 \\ 2 & 4 \\ 1 & 2\end{array}$

55

101

Phase 4

$13 \quad 23$

$14 \quad 25$

$20 \quad 35$

$\begin{array}{lr}9 & 16 \\ 1 & 1\end{array}$

49

111

2
48

111

$11 \quad 20$

$13 \quad 24$

$10 \quad 19$

$10 \quad 19$

35
1

18

20

$7 \quad 15$

$8 \cdot 17$

$20 \quad 43$

$\begin{array}{ll}8 & 17\end{array}$

11

$\begin{array}{ll}1 & 1 \\ 1 & 1\end{array}$

$\begin{array}{ll}1 & 1 \\ 1 & 1\end{array}$

24

24

$\begin{array}{ll}1 & 1 \\ 2 & 4\end{array}$

2

54

Phase 5

1241 $\begin{array}{rr}57 & 54 \\ 93 & 104\end{array}$
Table 1 Continued

\begin{tabular}{|c|c|c|c|c|c|c|}
\hline \multirow{2}{*}{$\begin{array}{c}\text { Lick } \\
\text { Burst } \\
\text { Lengths }\end{array}$} & \multicolumn{4}{|c|}{ Groups } & \multicolumn{2}{|c|}{3} \\
\hline & $\mathrm{N}$ & $\begin{array}{l}\text { Per- } \\
\text { cent }\end{array}$ & $\mathbf{N}$ & $\begin{array}{l}\text { Per- } \\
\text { cent }\end{array}$ & $\mathbf{N}$ & $\begin{array}{l}\text { Per- } \\
\text { cent }\end{array}$ \\
\hline $46-90$ & 13 & 24 & 6 & 20 & 5 & 28 \\
\hline $91-135$ & 8 & 10 & 4 & 14 & 2 & 10 \\
\hline $136-180$ & 3 & 4 & 2 & 8 & 1 & 6 \\
\hline $181-225$ & & & 1 & 3 & 1 & 6 \\
\hline $226-270$ & & & 1 & 3 & & \\
\hline $271-315$ & & & 1 & 3 & & \\
\hline
\end{tabular}

316-360

$361-405$

406-450

$\begin{array}{lrrrrrr}\text { Over } 450 & 1 & 3 & 2 & 8 & 1 & 6\end{array}$

$\begin{array}{llll}\text { Total } & 76 & 29 & 18 \\ \text { Median Length } & 34 & 60 & 54\end{array}$

Note $-G P 1=2.0-m A$ shock, $G P 2=1.0-m A$ shock, $G P 3=.1-m A$ shock.

the highest percentage of longer lick bursts occurred under the first baseline condition (Phase 1). Under the SIP contingencies in Phase 2, Group 1 subjects initiated the lick burst with the same frequency as in the first phase, but the median length of the burst increased an average of 2.5 times for each subject, indicating that while licking as often during baseline, they were also licking for a longer period of time. The subsequent introduction of lick-contingent shock $(2.0-\mathrm{mA})$ in Phase 3 showed a definite effect in which, generally, all subjects initiated licking 1.4 times more often than in Phase 2, but the disruptive effect of the shock variable resulted in a shortening of the median lick burst lengths to $32 \%$ of what they were without shock. When the shock contingency was discontinued in Phase 4, all subjects initiated licking more often than before, and the median length of the lick bursts generally returned to the levels present in Phases 1 and 5. Apparently the motivation to initiate licking in Phase 4 was stronger than in other phases and the removal of the shock contingency eliminated the disruption of drinking due to 4 the presentation of shock. Phase 5 lick burst initiation frequencies were slightly higher than in Phase 1 for all subjects, but the durations of the bursts tended to equal or fall below the durations exhibited in Phase 1. This appears to account for the fact that the Group 1 mean water intakes for both Phase 5 and Phase 1 were nearly equivalent.

With the instigation of SIP contingencies in Phase 2, all Group 2 subjects (1.0-mA shock) increased the median lengths of their lick bursts over Phase 1 baseline. During the Phase 3 shock condition the number of lick burst initiations were slightly reduced, but the bursts tended to be longer than before. This trend of fewer lick burst initiations but longer lick bursts did not significantly alter group mean water intake. This same general pattern of drinking was exhibited in Phase 4 by all subjects, indicating a conditioned reaction may have 
been established in the previous shock phase. Unlike Group 1, however, the shock received by Group 2 (1.0-mA) was apparently not so punishing as to seriously disrupt on-going drinking behavior, so there was no dramatic gain in subsequent mean water intake once the shock was discontinued. It is important to note, however, that the conditioned emotional response was sufficient to maintain a high level of drinking in a situation where decreases in the behavior would otherwise be expected. Phase 5 saw a reduction in both lick initiation and burst length, and mean water intuhes for both Phase 5 and Phase 1 were nearly equivalent.

Group 3 subjects (.1-mA shock) had the longest lick bursts occur in Phases 2 and 3. Once Phase 1 baseline had been established and Phase 2 was begun, all subjects tended to increase the frequency of lick burst initiation by a factor of three with median lick burst durations about five times as long. The total effect of introducing the shock contingency $(.1-\mathrm{mA})$ in Phase 3 was an increase in mean water intake for the group, accounted for by changes in individual subject lick burst parameters which appeared to vary in a nonsystematic fashion. Cessation of shock in the following phase showed a fairly uniform trend for all subjects in that the frequency of lick burst initiations increased slightly while the median lick burst length remained approximately the same. Group 3 mean water intake for Phase 4 was slightly higher than that of Phase 2, which may be explained by considering that schedule-induced drinking as an emotionally induced response was further increased by the introduction of shock in Phase 3 which served to increase still further emotional arousal and subsequent drinking. Lick burst patterns for Group 3 in Phase 5 showed equal lick burst initiations but longer median lick burst length when compared to those in Phase 1.

The theory suggesting that polydipsia is essentially a stress-induced behavior related to underlying emotional arousal was generally upheld. The effects of mild footshock (.1-mA) upon animals already under intense emotional arousal due to intermittent food scheduling and food deprivation was found to be facilitative to drinking as has been reported in other studies (Segal \& Oden, 1969). The effects of more punishing levels of shock were found to have no influence $(1.0-\mathrm{mA})$ or to disrupt drinking $(2.0-\mathrm{mA})$. Interestingly enough, the effect was sufficient to carry across the shock phase and significantly affect subsequent nonshocked drinking apparently due to some emotional condition from the pairing of shock with the SIP situation.

The foregoing experimental results raise several interesting points regarding punishment and its effects in the modification of behavior. It seems from the results of this study that the effects of punishment may differ substantially depending on the degree of intensity or severity of the punishment employed. Lower intensity levels of punishment have been shown to augment rather than decrease some behaviors (Segal \& Oden, 1969), specifically those behaviors seeming to be the most probable following reinforcement under emotional arousal conditions (King, 1974). Moderate levels of punishment, contrary to expectations, may have little or no significant disruptive effects when applied to behaviors which at least in part are motivated by emotional arousal. In fact, moderate levels of punishment may actually serve to extend the life of an emotionally-induced behavior beyond its usual expectancy. Higher levels of punishment as investigated in this study may be effective in suppressing behavior, but this effect was apparently lost once the punishing contingency was removed. In the present experiment, the high intensity shock group $(2.0-\mathrm{mA})$ significantly reduced drinking levels during the SIP plus shock phase. Once the shock was discontinued, however, previous levels of drinking were not only regained but actually surpassed.

As far as the results of the present study utilizing infrahuman subjects can be applied to human problems, such dramatic recovery of previously punished response modes may be of special interest to therapists who attempt to eliminate undesirable emotionally induced behaviors in the clients with whom they work. First, any therapeutic approach which utilizes punishment techniques must be carefully considered in terms of the intensity of the punishing stimulus to be employed. Although perhaps limited in scope, the data presented here suggest that a punishing stimulus of insufficient intensity will either have no effect or it may even facilitate the occurrence of an emotionally-induced undesirable behavior. Second, the data suggest that any therapeutic approach which uses punishment as the primary or exclusive treatment modality may result in an actual increase in the rate of the undesirable behavior when the punishing stimulus is discontinued. The results of the present experiment serve to underscore the need for concurrent positive reinforcement for establishing alternative behaviors which are desirable. This consideration alludes to a possibility that exists for further investigation in which an alternative response to drinking rewarded in conjunction with the punishment of the drinking response by an intense stimulus may lead to the subsequent substitution of that alternative response for drinking in the schedule-induced polydipsia paradigm.

\section{REFERENCES}

Calvin, A. D., \& Behan, R. A. The effect of hunger upon drinking in the rat. British Journal of Psychology, 1954, 45, 294-298.

Christian, W. P., \& Schaeffer, R. W. Advisability of using sugar-free food pellets for schedule-induced polydipsia research. Psychological Reports, 1973, 32, 274.

Falk, J. L. Production of polydipsia in normal rats by an intermittent food schedule. Science, 1961, 133, 195-196.

Falk, J. L. Studies on schedule-induced polydipsia. In M. J. Wayner (Ed.), Thirst. New York: Macmillan Company, 1964.

King, G. D. Three experiments on stress-induced behaviors. Unpublished doctoral dissertation, Florida State University, 1972.

King, G. D. The enhancement of schedule-induced polydipsia by preschedule noncontingent shock. Bulletin of the Psychonomic Society, 1974, 3, 46-48.

Levine, S. Water consumption: Emotionally produced facilitation or suppression? Psychonomic Science, 1965, 3, 105-106.

Moyer, K. E. Effects of experience with emotion provoking stimuli on water consumption in the rat. Psychonomic Science, 1965, 2, 251-252.

Roll, D., Schaeffer, R. W., \& Smith, J. C. Effects of a conditioned taste aversion on schedule-induced polydipsia. Psychonomic Science, 1969, 16, 39-40.

Segal, E. F., \& Oden, D. L. Determinants of polydipsia in rats: A reply to Stein. I. Emptying the water bottle. Psychonomic Science, 1965, 2, 201-202.

Segal, E. F., \& Oden, D. L. Effects of drinkometer current and of foot shock on psychogenic polydipsia. Psychonomic Science, 1969, 14, 13-15.

(Received for publication October 29, 1974.) 